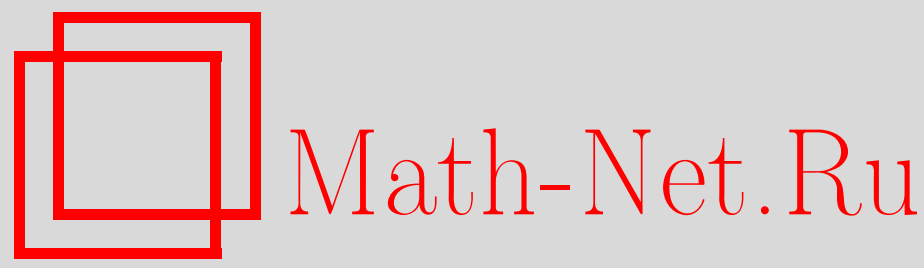

В. В. Сенатов, Об асимптотических разложениях в центральной предельной теореме с явными оценками остаточных членов, Теория вероятн. и ее примен., 2006, том 51, выпуск 4, 810-816

DOI: https://doi.org/10.4213/tvp29

Использование Общероссийского математического портала Math-Net.Ru подразумевает, что вы прочитали и согласны с пользовательским соглашением

http://www . mathnet.ru/rus/agreement

Параметры загрузки:

IP: 3.85 .5 .30

26 апреля 2023 г., 14:28:54 
(C) $2006 \mathrm{r}$.

СЕНАТОВ В. В.*

\section{ОБ АСИМПТОТИЧЕСКИХ РАЗЛОЖЕНИЯХ В ЦЕНТРАЛЬНОЙ ПРЕДЕЛЬНОЙ ТЕОРЕМЕ С ЯВНЫМИ ОЦЕНКАМИ ОСТАТОЧНЫХ ЧЛЕНОВ ${ }^{1)}$}

В статье приводятся явные оценки точности, которые гарантируют асимптотические разложения в центральной предельной теореме.

Ключевые слова и фразы: центральная предельная теорема, асимптотические разложения, точность аппроксимации.

Основная цель данной работы - продемонстрировать некоторые асимптотические разложения в центральной предельной теореме (ЦТП) с явными оценками точности, которую они гарантируют.

Пусть $X_{1}, X_{2}, \ldots$ - независимые одинаково распределенные случайные величины с нулевым средним и единичной дисперсией. Обозначим $F$ их общую функцию распределения, $f$ - соответствующую характеристическую функцию, $\Phi-$ функцию распределения стандартного нормального закона и $\varphi(x)=(2 \pi)^{-1 / 2} e^{-x^{2} / 2}-$ ее плотность. Будем считать, что для некоторого целого числа $m$ абсолютный момент $\beta_{m+2}=\mathbf{E}\left|X_{1}\right|^{m+2}$ конечен. Мы приведем примеры асимптотических разложений распределений нормированных сумм $\left(X_{1}+\cdots+X_{n}\right) / \sqrt{n}$ как для гладких, так и для решетчатых функций распределения $F$ исходных случайных величин $X_{1}, X_{2}, \ldots$, при этом гладкими мы будем называть распределения $F$ такие, что для некоторого числа $\nu>0$

$$
\int_{-\infty}^{\infty}|f(t)|^{\nu} d t<\infty
$$

Результаты, сформулированные ниже, дают асимптотические разложения в ЦПТ для плотностей гладких распределений и для функций распределения решетчатых распределений. Результаты, относящиеся к асимптотическим разложениям для функций распределения гладких распределений и к асимптотическим разложениям в локальной ЦТП для решетчатых распределений, легко получаются из формул, дающих асимптотические разложения для плотностей гладких распределений, и здесь мы их не приводим.

Обозначим $F_{n}$ функцию распределения нормированной суммы $\left(X_{1}+\cdots+X_{n}\right) / \sqrt{n}$,

$$
F_{n}(x)=\mathbf{P}\left\{\frac{X_{1}+\cdots+X_{n}}{\sqrt{n}}<x\right\} .
$$

Выполнение условия (1) гарантирует сушествование непрерывной плотности $p_{n}$ функции распределения $F_{n}$ при $n \geqslant \nu$.

Для формулировки результатов нам понадобятся числа

$$
\theta_{k}=\int_{-\infty}^{\infty} H_{k}(x) d F(x), \quad k=0,1, \ldots, m+1,
$$

где $H_{k}(x)=(-1)^{k}\left(e^{-x^{2} / 2}\right)^{(k)} e^{x^{2} / 2}-$ многочлены Чебышёва-Эрмита. Нетрудно показать, что

$$
\frac{\theta_{k}}{k !}=\sum_{j=0}^{[k / 2]} \frac{\alpha_{k-2 j}}{(k-2 j) !} \frac{(-1)^{j}}{2^{j} j !}
$$

* Московский государственный университет им. М.В. Ломоносова, механикоматематический факультет, кафедра теории вероятностей, Ленинские горы, 119992 Москва, Россия; e-mail: semenova@greatbook.ru

1) Работа выполнена при поддержке РФФИ (гранты 02-01-00600, 05-01-00583). 
где $\alpha_{l}=\mathbf{E} X_{1}^{l}, l=0,1, \ldots, m+1,-$ (степенные) моменты функции распределения $F$. Числа $\theta_{k}$ мы будем называть моментами Чебышёва-Эрмита функции распределения $F$. Отметим, что $\theta_{0}=1$ для любой функции распределения, для распределений с нулевым средним и единичной дисперсией $\theta_{1}=\theta_{2}=0$, величина $\theta_{3}=\alpha_{3}$ совпадает с асимметрией, а $\theta_{4}=\alpha_{4}-3$ совпадает с эксцессом функции распределения $F$.

Нам понадобятся также числа $\left\|\theta_{m+2}\right\|$, которые получаются по формуле (2) при $k=m+2$, но для четных $m$ в (2) следует снять множители $(-1)^{j}$, а для нечетных $m$ в (2) следует заменить $\alpha_{k-2 j}$ для $j \geqslant 1$ на их абсолютные значения, снять множители $(-1)^{j}$ и вместо $\alpha_{m+2}$ использовать $\beta_{m+2}$. Через $\left\|\theta_{m+3}^{(m+1)}\right\|$ мы будем обозначать числа, которые получаются по формуле (2) при $k=m+3$, в которой следует заменить $\alpha_{k-2 j}, j \geqslant 1$, на их абсолютные значения, снять множители $(-1)^{j}$, а слагаемое, формально связанное с моментом $\alpha_{m+3}$, - опустить.

Помимо только что определенных величин нам понадобятся числа $B_{k, n}$ и $B_{k, n-1}$, $k=4,5, \ldots$ :

$$
B_{k, n}=\frac{1}{2 \pi} \int_{-T \sqrt{n}}^{T \sqrt{n}}|t|^{k} \mu^{n}\left(\frac{t}{\sqrt{n}}\right) d t, \quad B_{k, n-1}=\frac{1}{2 \pi} \int_{-T \sqrt{n}}^{T \sqrt{n}}|t|^{k} \mu^{n-1}\left(\frac{t}{\sqrt{n}}\right) d t,
$$

где функция $\mu(t) \geqslant e^{-t^{2} / 2}$ и число $T>0$ таковы, что

$$
|f(t)| \leqslant \mu(t) \quad \text { при }|t| \leqslant T .
$$

Такая пара $(\mu, T)$ для заданной характеристической функции $f$ сушествует всегда, и при достаточно широких условиях на функцию распределения $F$ функцию $\mu$ и число $T$ можно выбрать так, что

$$
B_{k, n} \rightarrow B_{k} \quad \text { и } \quad B_{k, n-1} \rightarrow B_{k} \quad \text { при } \quad n \rightarrow \infty, k=4,5, \ldots,
$$

где $B_{k}$ - абсолютный момент $k$-го порядка стандартного нормального закона, деленный на $\sqrt{2 \pi}$. Например, для распределений с $\alpha_{3}=0$ и $\theta_{4}<3$ в качестве функции $\mu(t)$ можно взять $\left(1+t^{2} / 2\right)^{-1}$ и в качестве числа $T$ можно взять $\sqrt{2 \min \left(1,\left(3-\theta_{4}\right) /\left(3+\theta_{4}\right)\right)}$. При этом, в частности, для нечетных $k, k<2 n-1$,

$$
B_{k, n} \leqslant B_{k}\left[\left(1-\frac{1}{n}\right) \cdots\left(1-\frac{k+1}{2 n}\right)\right]^{-1}
$$

Наконец отметим, что при выполнении условия (1) величина

$$
\alpha(T)=\max \{|f(t)|: t \geqslant T\}
$$

строго меньше 1 для любого $T>0$. В теоремах 2 и 3 нам понадобится величина $\beta(T)$, которая определяется аналогично величине $\alpha(T)$ :

$$
\beta(T)=\max \left\{e^{-t^{2} / 2}\left|1+\frac{\theta_{4}}{4 !} t^{4}\right|: t \geqslant T\right\}
$$

и которая также строго меньше 1 , если, скажем, $\theta_{4} \leqslant 6$. В этих же теоремах мы будем предполагать, что пара $(\mu, T)$ такова, что

$$
e^{-t^{2} / 2}\left|1+\frac{\theta_{4}}{4 !} t^{4}\right| \leqslant \mu(t) \quad \text { при } \quad|t| \leqslant T
$$

В приведенном выше примере пары $(\mu, T)$ выполнение этого условия следует из выполнения условия (3).

Теорема 1. Пусть четвертый момент Функиии распределения $F$ конечен, выполнено условие (1) и для некоторой пары $(\mu, T)$ выполнено условие (3). Тогда для любого $n \geqslant \max (\nu, 2)$ и всех действительных $x$

$$
p_{n}(x)=\varphi(x)+\frac{\theta_{3}}{3 !} \frac{1}{\sqrt{n}} H_{3}(x) \varphi(x)+R
$$


¿de

$$
\begin{aligned}
|R| \leqslant & \left(\frac{\beta_{4}}{4 !}+\frac{1}{8}\right) \frac{B_{4, n-1}}{n}+\frac{1}{2}\left(\frac{\theta_{3}}{3 !}\right)^{2} \frac{B_{6}}{n}+\frac{\left\|\theta_{5}^{(3)}\right\|}{5 !} \frac{B_{5, n-1}}{n^{3 / 2}} \\
& +\frac{1}{2}\left(\frac{\theta_{3}}{3 !}\right)^{2} \frac{B_{6, n}}{n^{2}}+\frac{\sqrt{n}}{\pi} \alpha^{n-\nu}(T) \int_{T}^{\infty}|f(t)|^{\nu} d t+\frac{1}{\pi T \sqrt{n}} e^{-T^{2} n / 2}
\end{aligned}
$$

Теорема 2. Пусть пятый момент функиии распределения $F$ конечен, величина $\theta_{4}<6$ и для некоторой пары $(\mu, T)$ выполнены условия (3) и (4). Тогда для $n \geqslant$ $\max (\nu, 2)$ makux, umo

и всех действительных $x$

$$
\rho=\frac{\left|\theta_{4}\right|}{6}+\frac{\left|\theta_{4}\right|}{4 ! n}<1
$$

$$
p_{n}(x)=\varphi(x)+\frac{\theta_{3}}{3 !} \frac{1}{\sqrt{n}} H_{3}(x) \varphi(x)+\frac{\theta_{4}}{4 !} \frac{1}{n} H_{4}(x) \varphi(x)+\frac{1}{2}\left(\frac{\theta_{3}}{3 !}\right)^{2} \frac{1}{n} H_{6}(x) \varphi(x)+R,
$$

гde

$$
\begin{aligned}
|R| \leqslant & \frac{\left\|\theta_{5}\right\|}{5 !} \frac{B_{5, n-1}}{n^{3 / 2}}+\frac{n+1}{n} \frac{\left|\theta_{3}\right|}{3 !} \frac{\left|\theta_{4}\right|}{4 !} \frac{B_{7, n}}{n^{3 / 2}}+\frac{1}{3 !}\left|\frac{\theta_{3}}{3 !}\right|^{3} \frac{B_{9}}{n^{3 / 2}} \\
& +\frac{\left\|\theta_{6}^{(4)}\right\|}{6 !} \frac{B_{6, n-1}}{n^{2}}+\frac{1}{2}\left(\frac{\theta_{3}}{3 !}\right)^{2} \frac{B_{6, n}}{n^{2}}+\frac{1}{1-\rho} \frac{1}{2}\left(\frac{\theta_{4}}{4 !}\right)^{2} \frac{B_{8}}{n^{2}} \\
& +\frac{\sqrt{n}}{\pi} \alpha^{n-\nu}(T) \int_{T}^{\infty}|f(t)|^{\nu} d t+\frac{\sqrt{n}}{\pi} \beta^{n-1}(T) \int_{T}^{\infty} e^{-t^{2} / 2}\left|1+\frac{\theta_{4}}{4 !} t^{4}\right| d t .
\end{aligned}
$$

В этой теореме условия, связанные с величиной $\theta_{4}$, можно снять, при этом оценка остаточной части разложения станет несколько более громоздкой.

Теорема 3. Пусть шестой момент функиии распределения $F$ конечен и выполнены все условия теоремы 2. Тогда для тех же $n$, что и в теореме 2 , и всех действительных $x$

$$
\begin{aligned}
p_{n}(x)= & \varphi(x)+\frac{\theta_{3}}{3 !} \frac{1}{\sqrt{n}} H_{3}(x) \varphi(x)+\frac{\theta_{4}}{4 !} \frac{1}{n} H_{4}(x) \varphi(x) \\
& +\frac{1}{2}\left(\frac{\theta_{3}}{3 !}\right)^{2} \frac{1}{n} H_{6}(x) \varphi(x)+\frac{\theta_{5}}{5 !} \frac{1}{n^{3 / 2}} H_{5}(x) \varphi(x) \\
& +\frac{\theta_{3}}{3 !} \frac{\theta_{4}}{4 !} \frac{1}{n^{3 / 2}} H_{7}(x) \varphi(x)+\frac{1}{3 !}\left(\frac{\theta_{3}}{3 !}\right)^{3} \frac{1}{n^{3 / 2}} H_{9}(x) \varphi(x)+R
\end{aligned}
$$

วde

$$
\begin{aligned}
|R| \leqslant & \frac{\left\|\theta_{6}\right\|}{6 !} \frac{B_{6, n-1}}{n^{2}}+\frac{1}{2}\left(\frac{\theta_{3}}{3 !}\right)^{2} \frac{B_{6, n}}{n^{2}}+\frac{\left|\theta_{3}\right|}{3 !} \frac{\left|\theta_{5}\right|}{5 !} \frac{B_{8}}{n^{2}}+\frac{1}{1-\rho} \frac{1}{2}\left(\frac{\theta_{4}}{4 !}\right)^{2} \frac{B_{8}}{n^{2}} \\
& +\frac{1}{2}\left(\frac{\theta_{3}}{3 !}\right)^{2} \frac{\left|\theta_{4}\right|}{4 !} \frac{B_{10}}{n^{2}}+\frac{1}{4 !}\left(\frac{\theta_{3}}{3 !}\right)^{4} \frac{B_{12}}{n^{2}}+\frac{\left\|\theta_{7}^{(5)}\right\|}{7 !} \frac{B_{7, n-1}}{n^{5 / 2}} \\
& +\frac{\left|\theta_{3}\right|}{3 !} \frac{\left|\theta_{4}\right|}{4 !} \frac{B_{7, n}}{n^{5 / 2}}+\frac{n+1}{n} \frac{\left|\theta_{4}\right|}{4 !} \frac{\left|\theta_{5}\right|}{5 !} \frac{B_{9, n}}{n^{5 / 2}}+\frac{\left|\theta_{3}\right|}{3 !} \frac{\left|\theta_{5}\right|}{5 !} \frac{B_{8, n}}{n^{3}}+\frac{n+1}{2 n}\left(\frac{\theta_{5}}{5 !}\right)^{2} \frac{B_{10}, n}{n^{3}} \\
& +\frac{\sqrt{n}}{\pi} \alpha^{n-\nu}(T) \int_{T}^{\infty}|\rho(t)|^{\nu} d t+\frac{\sqrt{n}}{\pi} \beta^{n-1}(T) \int_{T}^{\infty} e^{-t^{2} / 2}\left|1+\frac{\theta_{4}}{4 !} t^{4}\right| d t .
\end{aligned}
$$

Суммы величин, участвующих в правых частях разложений в теоремах 1-3, за исключением величин $R$, мы будем называть главными частями асимптотических разложений, а величины $R$ - остаточными частями разложений.

Разложения, приведенные в теоремах 1-3, мы будем называть разложениями Эджворта-Крамера, поскольку все слагаемые в главных частях этих разложений при росте $n$ убывают медленнее остаточных частей разложений, которые стремятся к нулю как $1 / n^{m / 2}, m=2,3$ и 4 в теоремах 1,2 и 3 соответственно, и в главных 
частях разложений используется информация лишь о моментах исходного распределения, порядки которых не превосходят $m+1$.

Обратим внимание на то, что в оценках остаточных частей разложений, приведенных в теоремах 1-3, несколько слагаемых убывает при росте $n$ как $1 / n^{m / 2}$. Для разложений Эджворта-Крамера это связано с существом дела, но можно предложить асимптотические разложения, в оценках остаточных частей которых лишь одно слагаемое убывает при росте $n$ как $1 / n^{m / 2}$.

Такие модификации разложений Эджворта-Крамера мы будем называть уточненными разложениями Эджворта-Крамера. Пример такого разложения дает следующая теорема.

Теорема 4. При выполнении условий теоремы 2 для тех же $n$, что и в теореме 2 , и всех действительных $x$

$$
\begin{aligned}
p_{n}(x)= & \varphi(x)+\frac{\theta_{3}}{3 !} \frac{1}{\sqrt{n}} H_{3}(x) \varphi(x)+\frac{\theta_{4}}{4 !} \frac{1}{n} H_{4}(x) \varphi(x) \\
& +\frac{1}{2}\left(\frac{\theta_{3}}{3 !}\right)^{2} \frac{1}{n} H_{6}(x) \varphi(x)+\frac{\theta_{3}}{3 !} \frac{\theta_{4}}{4 !} \frac{1}{n^{3 / 2}} H_{7}(x) \varphi(x) \\
& +\frac{1}{3 !}\left(\frac{\theta_{3}}{3 !}\right)^{3} \frac{1}{n^{3 / 2}} H_{9}(x) \varphi(x)+R,
\end{aligned}
$$

¿de

$$
\begin{aligned}
|R| \leqslant & \frac{\left\|\theta_{5}\right\|}{5 !} \frac{B_{5, n-1}}{n^{3 / 2}}+\frac{\left\|\theta_{6}^{(4)}\right\|}{6 !} \frac{B_{6, n-1}}{n^{2}}+\frac{1}{2}\left(\frac{\theta_{3}}{3 !}\right)^{2} \frac{B_{6, n}}{n^{2}}+\frac{1}{1-\rho} \frac{1}{2}\left(\frac{\theta_{4}}{4 !}\right)^{2} \frac{B_{8}}{n^{2}} \\
& +\frac{1}{2}\left(\frac{\theta_{3}}{3 !}\right)^{2} \frac{\left|\theta_{4}\right|}{4 !} \frac{B_{10}}{n^{2}}+\frac{1}{4 !}\left(\frac{\theta_{3}}{3 !}\right)^{4} \frac{B_{12}}{n^{2}}+\frac{\left|\theta_{3}\right|}{3 !} \frac{\left|\theta_{4}\right|}{4 !} \frac{B_{7, n}}{n^{5 / 2}} \\
& +\frac{\sqrt{n}}{\pi} \alpha^{n-\nu}(T) \int_{T}^{\infty}|f(t)|^{\nu} d t+\frac{\sqrt{n}}{\pi} \beta^{n-1}(T) \int_{T}^{\infty} e^{-t^{2} / 2}\left|1+\frac{\theta_{4}}{4 !} t^{4}\right| d t .
\end{aligned}
$$

Разложения Эджворта-Крамера, продемонстрированные в теоремах 1-3 (при условии, что момент $\beta_{m+2}, m=2,3$, и 4 , конечен), можно получать и при больших значениях $m$. При этом оказывается, что остаточные части разложений убывают при росте $n$ как $1 / n^{m / 2}$, а в главные части этих разложений необходимо включать функции $H_{l} \varphi \mathrm{c} l=3,4, \ldots, 3 m-3, l \neq 3 m-4$, с некоторыми коэффициентами, зависящими от распределения исходных случайных величин $X_{1}, X_{2}, \ldots$ и от числа слагаемых в нормированных суммах этих величин. Для каждого фиксированного $l>5$ коэффициенты при $H_{l} \varphi$ при увеличении $m$ изменяются до тех пор, пока они не станут равными $\theta_{l}\left(F_{n}\right) / l$ ! , где $\theta_{l}\left(F_{n}\right)$ - момент Чебышёва-Эрмита порядка $l$ функции распределения $F_{n}$. Эти величины вычисляются по формулам

$$
\frac{\theta_{l}\left(F_{n}\right)}{l !}=\sum \frac{n !}{k_{0} ! k_{3} ! \cdots k_{l} !}\left(\frac{\theta_{3}}{3 ! n^{3 / 2}}\right)^{k_{3}} \cdots\left(\frac{\theta_{l}}{l ! n^{l / 2}}\right)^{k_{l}},
$$

где $\theta_{3}, \ldots, \theta_{l}$ - моменты Чебышёва-Эрмита функции распределения $F$, а суммирование проводится по всем наборам неотрицательных целых чисел $k_{0}, k_{3}, \ldots, k_{l}$ таким, что

$$
k_{0}+k_{3}+\cdots+k_{l}=n, \quad 3 k_{3}+\cdots+l k_{l}=l .
$$

Попытки получить при условии конечности момента $\beta_{m+2}, m \geqslant 3$, асимптотические разложения, остаточные части которых с ростом $n$ убывают как $1 / n^{m / 2}$ и в которых коэффициенты при $H_{l} \varphi, l=3, \ldots, 3 m-3, l \neq 3 m-4$, с самого начала совпадают с $\theta_{l}\left(F_{n}\right) / l$ !, не могут привести к успеху, поскольку при этом понадобится информация о моментах функции распределения $F$ вплоть до порядка $3 m-3>m+2$. Однако можно получать асимптотические разложения, в которых коэффициенты при $H_{l} \varphi, l=3,4, \ldots, m+1$, совпадают с $\theta_{l}\left(F_{n}\right) / l !$, а коэффициенты при $H_{l} \varphi, l=m+2, \ldots, 3 m-3, l \neq 3 m-4$, связаны с так называемыми квазимоментами Чебышёва-Эрмита $\theta_{l}^{(m+1)}\left(F_{n}\right)$ (уровня $m+1$ ) распределения $F_{n}$, которые получаются по той же формуле, что и $\theta_{l}\left(F_{n}\right)$, в которой следует опустить слагаемые, 
связанные с $\theta_{j}, j>m+1$. Так получаются асимптотические разложения, которые мы называем короткими разложениями Грама-Шарлье. Пример такого разложения дает следуюшая теорема.

Теорема 5. Пусть выполнены условия теоремы 2. Тогда для тех же п, что и в теореме 2, и всех действительных $x$

$p_{n}(x)=\varphi(x)+\frac{\theta_{3}}{3 !} \frac{1}{\sqrt{n}} H_{3}(x) \varphi(x)+\frac{\theta_{4}}{4 !} \frac{1}{n} H_{4}(x) \varphi(x)+\frac{n-1}{2 n}\left(\frac{\theta_{3}}{3 !}\right)^{2} \frac{1}{n} H_{6}(x) \varphi(x)+R$,

гde

$$
\begin{aligned}
|R| \leqslant & \frac{\left\|\theta_{5}\right\|}{5 !} \frac{B_{5, n-1}}{n^{3 / 2}}+\frac{\left|\theta_{3}\right|}{3 !} \frac{\left|\theta_{4}\right|}{4 !} \frac{B_{7}}{n^{3 / 2}}+\frac{1}{3 !}\left|\frac{\theta_{3}}{3 !}\right|^{3} \frac{B_{9}}{n^{3 / 2}}+\frac{\left\|\theta_{6}^{(4)}\right\|}{6 !} \frac{B_{6, n-1}}{n^{2}} \\
& +\frac{1}{1-\rho} \frac{1}{2}\left(\frac{\theta_{4}}{4 !}\right)^{2} \frac{B_{8}}{n^{2}}+\frac{\left|\theta_{3}\right|}{3 !} \frac{\left|\theta_{4}\right|}{4 !} \frac{B_{7, n}}{n^{5 / 2}}+\frac{1}{2}\left|\frac{\theta_{3}}{3 !}\right|^{3} \frac{B_{9, n}}{n^{5 / 2}} \\
& +\frac{1}{2}\left(\frac{\theta_{3}}{3 !}\right)^{2} \frac{\left\|\theta_{4}\right\|}{4 !} \frac{B_{10, n-1}}{n^{3}}+\frac{1}{3 !}\left|\frac{\theta_{3}}{3 !}\right|^{3} \frac{B_{9, n}}{n^{7 / 2}}+\frac{1}{4}\left(\frac{\theta_{3}}{3 !}\right)^{2} \frac{\left\|\theta_{5}^{(3)}\right\|}{5 !} \frac{B_{11, n-1}}{n^{7 / 2}} \\
& +\frac{1}{8}\left(\frac{\theta_{3}}{3 !}\right)^{4} \frac{B_{12, n}}{n^{4}}+\frac{\sqrt{n}}{\pi} \alpha^{n-\nu}(T) \int_{T}^{\infty}|f(t)|^{\nu} d t \\
& +\frac{\sqrt{n}}{\pi} \beta^{n-1}(T) \int_{T}^{\infty} e^{-t^{2} / 2}\left|1+\frac{\theta_{4}}{4 !} t^{4}\right| d t+\frac{1}{2}\left(\frac{\theta_{3}}{3 !}\right)^{2} \frac{1}{n^{2}} L_{6}(T \sqrt{n}),
\end{aligned}
$$

$a$

$$
L_{6}(T \sqrt{n})=\frac{1}{\pi} \int_{T \sqrt{n}}^{\infty} t^{6} e^{-t^{2} / 2} d t .
$$

В коротких разложениях Грама-Шарлье, как и в разложениях ЭджвортаКрамера, оценки остаточных частей разложений содержат несколько слагаемых, убывающих с ростом $n$ как $1 / n^{m / 2}$. Можно получать разложения, оценки остаточных частей которых содержат лишь одно слагаемое $\left(\left\|\theta_{m+2}\right\| /(m+2) !\right) /\left(B_{m+2, n-1} / n^{m / 2}\right)$, убывающее при росте $n$ как $1 / n^{m / 2}$ (во всех оценках остаточных частей разложений, приводившихся выше, только это слагаемое связано с $\beta_{m+2}$ ). Такие разложения мы называем средними разложениями Грама-Шарлье, они аналогичны уточненным разложениям Эджворта-Крамера, и здесь мы их не приводим.

Дальнейшее развитие предложенных асимптотических разложений приводит $\mathrm{k}$ так называемым длинным разложениям Грама-Шарлье, в оценках остаточных частей которых величина $\left\|\theta_{m+2}\right\|$ заменяется на $\beta_{m+2}$. Этим оценкам можно придать вид

$$
|R| \leqslant \frac{\beta_{m+2}}{(m+2) !} \frac{B_{m+2}}{n^{m / 2}}\left(1+O\left(\frac{1}{\sqrt{n}}\right)\right), \quad n \rightarrow \infty .
$$

По-видимому, величина постоянной в этой оценке (по крайней мере, для четных $m$ ) неулучшаема. Для величины $O(1 / \sqrt{n})$ можно указать явную оценку.

Перейдем теперь к асимптотическим разложениям для функций распределения в случае решетчатых распределений.

Пусть функция распределения $F$ исходных случайных величин $X_{1}, X_{2}, \ldots$ решетчата с (максимальным) шагом $h>0$. Хорошо известно, что в этом случае точки роста функции распределения $F_{n}$ нормированной суммы $\left(X_{1}+\cdots+X_{n}\right) / \sqrt{n}$ принадлежат решетке $D_{n}=\left\{a_{n}+k h / \sqrt{n}: k=0, \pm 1, \pm 2, \ldots\right\}$. где $a_{n}$ - некоторое действительное число, $F_{n}(x)$ изменяется только скачками, точки скачков принадлежат $D_{n}$ и величина максимального среди этих скачков не меньше $c h / \sqrt{n}$, где $c>0$ - абсолютная постоянная. Поэтому равномерное расстояние между $F_{n}(x)$ и $\Phi(x)$ при росте $n$ не может убывать быстрее, чем $1 / \sqrt{n}$, ни при каких, сколь угодно сильных, ограничениях на моменты $F$. Однако оказывается, что для $x \in D_{n}+h /(2 \sqrt{n})$ (т.е., грубо говоря, для середин тех интервалов постоянства функции распределения $F_{n}$, в которых $F_{n}(x) \neq 0$ и $F_{n}(x) \neq 1$ и которые не содержат точек из $\left.D_{n}\right)$ точность аппроксимации $F_{n}(x)$ нормальным законом $\Phi(x)$ может быть выше $1 / \sqrt{n}$. В частности, если третий момент $\alpha_{3}$ равен нулю, то $F_{n}(x)-\Phi(x)=O(1 / n)$ при $n \rightarrow \infty$ для $x \in D_{n}+h /(2 \sqrt{n})$, если $\beta_{4}<\infty$. 
Такой результат в случае, когда $F$ - распределение Бернулли (т.е. имеет лишь две точки роста), был получен Я.В. Успенским в 1937 г. Здесь и далее мы обозначаем $D_{n}+h /(2 \sqrt{n})$ сдвиг множества $D_{n}$ на $h /(2 \sqrt{n})$.

Оказывается, что для $x \in D_{n}+h /(2 \sqrt{n})$ можно строить и асимптотические разложения функции распределения $F_{n}$ сколь угодно высокой точности, если у функции распределения $F$ существует достаточное число моментов. Мы сформулируем соответствуюший результат лишь для четных $m$. При этом нам понадобится величина

$$
\|\theta T\|_{3, m+1}=3 ! \sum_{k=3}^{m+1} \frac{\left|\theta_{k}\right|}{k !} T^{k-3}, \quad T>0
$$

и числа Бернулли, которые мы будем обозначать $\widetilde{B}_{2 k}, k=1,2, \ldots$.

Теорема 6. Пусть для некоторого челого числа $m \geqslant 4$ момент $\beta_{m+2}$ решетчатой функиии распределения $F$ с шагом $h>0$, нулевым средним и единичной дисперсией конечен. Пусть для некоторой пары $(\mu, T)$, где $0<T \leqslant \min (1, \pi / h)$, выполнено условие (3) и

$$
e^{-t^{2} / 2}\left|1+\sum_{k=3}^{m+1} \frac{\theta_{k}}{k !}(i t)^{k}\right| \leqslant \mu(t) \quad \text { nри всех } \quad|t| \leqslant T
$$

где $\theta_{3}, \ldots, \theta_{m+1}$ - моменты Чебышёва-Эрмита функиии распределения $F$. Пусть $\|\theta T\|_{3, m+1}<3$ и число $n \geqslant m+2$ таково, что

$$
\frac{\|\theta T\|_{3, m+1}}{3}+\frac{1}{n}\left(\frac{\|\theta T\|_{3, m+1}}{3 !}\right)^{2}\left(\frac{(m+1)^{3}}{2}+9(m+1)^{2}+27(m+1)+9\right)<1 .
$$

Тогда для любого $x \in D_{n}+h /(2 \sqrt{n})$, где $D_{n}-$ решетка, на которой сосредоточено распределение $F_{n}$, при четном $m$ справедливо равенство

$$
\begin{aligned}
F_{n}(x)= & \Phi(x)-\sum_{l=3}^{m+1} \frac{\theta_{l}\left(F_{n}\right)}{l !} H_{l-1}(x) \varphi(x)-\sum_{\substack{l=m+2, l \neq 3 m-1}}^{3 m} \frac{\theta_{l}^{(m+1)}\left(F_{n}\right)}{l !} H_{l-1}(x) \varphi(x) \\
+ & \sum_{j=0}^{m / 2-1} \frac{2\left(2^{m-1-2 j}-1\right)}{(m-2 j) !} \widetilde{B}_{m-2 j}\left(\frac{h}{2 \sqrt{n}}\right)^{m-2 j} H_{m-2 j-1}(x) \varphi(x) \\
+ & \sum_{j=1}^{m / 2-1} \frac{2\left(2^{m-1-2 j}-1\right)}{(m-2 j) !} \widetilde{B}_{m-2 j}\left(\frac{h}{2 \sqrt{n}}\right)^{m-2 j} \\
& \quad \times \sum_{\substack{l=3, l \neq 6 j-1}}^{6 j} \frac{\theta_{l}^{(m+1)}\left(F_{n}\right)}{l !} H_{l+m-2 j-1}(x) \varphi(x)+O\left(\frac{1}{n^{m / 2}}\right) .
\end{aligned}
$$

Мы не приводим здесь явную оценку величины $O\left(1 / n^{m / 2}\right)$ из-за ее громоздкости. Кроме того, для формулировки этой оценки требуется вводить дополнительные характеристики функции распределения $F$. Отметим лишь, что единственное слагаемое в оценке $O\left(1 / n^{m / 2}\right)$, которое при росте $n$ убывает как $1 / n^{m / 2}$, есть

$$
c(T, h) \frac{\left\|\theta_{m+2}\right\|}{(m+2) !} \frac{B_{m+2, n-1}}{n^{m / 2}},
$$

где $c(T, h)=T h /(2 \sin (T h / 2)) \leqslant \pi / 2$. Отметим также, что сумма

$$
\Phi(x)-\sum_{l=3}^{m+1} \frac{\theta_{l}\left(F_{n}\right)}{l !} H_{l-1}(x) \varphi(x)-\sum_{\substack{l=m+2, l \neq 3 m-1}}^{3 m} \frac{\theta_{l}^{(m+1)}\left(F_{n}\right)}{l !} H_{l-1}(x) \varphi(x)
$$

в главной части разложения $F_{n}(x)$ для решетчатых распределений совпадает с главной частью среднего разложения Грама-Шарлье $F_{n}(x),-\infty<x<\infty$, для гладких 
распределений. Для того чтобы величина (5) давала для нерешетчатой функции распределения $F(x)$ асимптотическое разложение $F_{n}(x)$ с точностью $O\left(1 / n^{m / 2}\right), n \rightarrow \infty$, достаточно выполнения условия Крамера

$$
\limsup _{t \rightarrow \infty}|f(t)|<1 \text {. }
$$

Для нечетных $m$ асимптотическое разложение и оценка его остаточной части выглядят аналогично, лишь в некоторых местах следует заменить $m$ на $m-1$. Мы не будем останавливаться на этом.

В теоремах 2-6 присутствуют ограничения $\theta_{4}<6$ и $\|\theta T\|_{3, m+1}<3$. Эти ограничения можно обойти, но мы также не останавливаемся на этом.

Один из промежуточных результатов, полученных при доказательстве теоремы 6 , позволяет доказать следующее утверждение.

Теорема 7. Пусть $F$ - решетчатая функиия распределения с шагом $h>0$, нулевым средним, единичной дисперсией и конечным четвертым моментом. Для любого $n \geqslant 2$, любого $x \in D_{n}+h /(2 \sqrt{n})$ и любого числа $0<\delta<\frac{1}{3}$ справедливо неравенство

$$
\begin{aligned}
\left|F_{n}(x)-\Phi(x)\right| \leqslant & \frac{\left|\alpha_{3}\right|}{6 \sqrt{2 \pi n}}\left(\frac{n}{n-1}\right)^{3 / 2}(1-\delta)^{-3 / 2}+\frac{\beta_{4}+3}{12 \pi n}\left(\frac{n}{n-1}\right)^{2}(1-\delta)^{-2} \\
& +\left(\frac{h}{\sqrt{n}}\right)^{2}\left(\frac{(1-\delta)^{-2}}{3 \pi}+\frac{1}{24 \sqrt{2 \pi e}}\right)+\alpha^{n}\left(\frac{1}{\pi} \log \left(\frac{\pi}{3} \frac{\beta_{3}}{\delta h}\right)+\frac{\pi}{6}\right) \\
& +\frac{1}{\pi}\left(\frac{\beta_{3}}{3 \delta \sqrt{n}}\right)^{2} e^{-9 \delta^{2} n /\left(2 \beta_{3}^{2}\right)}+\frac{1}{\pi^{3}}\left(\frac{h}{\sqrt{n}}\right)^{2} e^{-\pi^{2} n /\left(2 h^{2}\right)}
\end{aligned}
$$

где $\alpha_{3}-$ третий момент функиии распределения $F, \alpha=\max \left\{|f(t)|: 3 \delta / \beta_{3} \leqslant t \leqslant\right.$ $\pi / h\}<1$, a $f$ - характеристическая функция распределения $F$

Следствием теоремы 7 является неравенство

$$
\sup _{-\infty<x<\infty}\left|F_{n}(x)-\Phi(x)\right| \leqslant \frac{\left|\alpha_{3}\right|}{6 \sqrt{2 \pi n}}+\frac{1}{\sqrt{2 \pi}} \frac{h}{2 \sqrt{n}}+O\left(\frac{1}{n}\right), \quad n \rightarrow \infty .
$$

Для величины $O(1 / n)$ легко получить явную оценку. Отметим, что оба слагаемых в правой части последнего неравенства, убывающие при росте $n$ как $1 / \sqrt{n}$, являются правильными.

Отметим также, что если в левой части последнего неравенства снять знак верхней грани, то во второе слагаемое правой части можно добавить множитель, асимптотически (при $n \rightarrow \infty$ ) равный $e^{-x^{2} / 2}$.

Перечисленные результаты и ряд других, вместе с их доказательствами, содержатся в монографии «Џентральная предельная теорема: точность аппроксимации и асимптотические разложения», готовящейся к печати в издательстве «ТВП».

(C) $2006 \Gamma$.

PANG T.-X.*, LIN Z.-Y.*

\section{A NONCLASSICAL CHUNG-TYPE LAW OF THE ITERATED LOGARITHM FOR I.I.D. RANDOM VARIABLES ${ }^{1)}$}

Пусть $\left\{X, X_{n} ; n \geqslant 1\right\}$ - последовательность независимых одинаково распределенных случайных величин; положим $S_{n}=\sum_{i=1}^{n} X_{i}$. Мы определяем последовательность положительных констант $\{d(n), n \geqslant 1\}$,

* Department of Mathematics, Zhejiang University, Hangzhou 310028, China; e-mail: pangtianxiao@tom.com; zlin@zju.edu.cn

1) Project supported by NSFC (10571159). 